\title{
Kernos
}

Revue internationale et pluridisciplinaire de religion grecque antique

1 | 1988

Varia

\section{Aux origines de la cité-État : sôphrosynè sociale et politique religieuse nationale}

Ioannis Loucas

\section{OpenEdition}

Journals

\section{Édition électronique}

URL : http://journals.openedition.org/kernos/105

DOI : 10.4000/kernos.105

ISSN : 2034-7871

\section{Éditeur}

Centre international d'étude de la religion grecque antique

\section{Édition imprimée}

Date de publication : 1 janvier 1988

ISSN : 0776-3824

\section{Référence électronique}

Ioannis Loucas, «Aux origines de la cité-État: sôphrosynè sociale et politique religieuse nationale », Kernos [En ligne], 1 | 1988, mis en ligne le 31 janvier 2011, consulté le 30 avril 2019. URL : http:// journals.openedition.org/kernos/105; DOI : 10.4000/kernos.105 


\section{AUX ORIGINES DE LA CITÉ-ÉTAT : SôPHROSYNÈ SOCIALE ET POLITIQUE RELIGIEUSE NATIONALE \\ (avec une référence spéciale à Athènes)}

Lorsque le rideau de l'histoire se lève sur la Grèce après l' «âge obscur» qui a suivi la prétendue invasion dorienne, les rois toutpuissants de l'épopée homérique ne sont plus que des magistrats religieux de rang secondaire ${ }^{1}$. Les Grecs, divisés en un grand nombre d'unités politiques autonomes et indépendantes, posent les fondements de la cité-Etat établie sur un nouvel ordre des choses dont les origines sont situées aux temps archaïques. Pour A. Hauser $^{2}$, l'époque archaiqque est le moment de l'instauration d'une nouvelle éthique qui repose sur une triple conception :

L'éphébie, soit la bonne condition physique et la discipline militaire fondée sur la tradition, la naissance et la race; la kalo(s)kagathia dont l'idéal est un équilibre entre le corporel et le spirituel, les qualités physiques et morales; la sôphrosynè qui prône l'empire sur soi, la maitrise et la modération.

Cependant, il paraît plus vraisemblable que seule la sôphrosynè soit la conception fondamentale, car les principes de l'éphébie et de la kalo(s)kagathia émanent directement d'elle - ou, plus exactement, ne sont que son application aux domaines de la guerre et de la morale $^{3}$. La signification du mot sôphrosyne englobe plusieurs notions, comme conception complete, sagesse, prudence, circonspection, modestie, pureté, sobriété, modération, contrôle de soi ${ }^{4}$ :

1 Cf. d'une façon générale, G. GLOTZ, La cité grecque, Paris, 1968, p. 118.

2 A. HAUSER, Histoire sociale de l'art et de la littérature, I : la Préhistoire et le Moyen Age, Paris, 1982, p. 84.

3 Sur la sôphrosynè, cf. en demier lieu J.M. HURWIT, The Art and Culture of Early Greece. 1100-480 B.C., New York, 1985, p. 344.

4 Outre les entrées $\sigma \omega ́ \varphi \rho \omega v$ et $\sigma \omega \varphi \rho o \sigma u ́ v \eta$ dans les dictionnaires de la langue grecque de A. Bailly et de H.G. Liddell, R. Scott et H.S. Jones, cf. entre autres H.D.F. KITTO, The Greeks, Harmondsworth, 1951, trad. en grec par I. Lampsas, Athènes, 1962, p. 210; H. NORTH, Sophrosyne. Self-Knowledge and Self-Restraint in Greek Literature, New York, 1966, p. 28-31. 
ce sont les notions dont il est toujours question lorsqu'on se réfère aux qualités morales et spirituelles qui ont étayé ce monument de la «mesure» et de l' «harmonie» qu'a offert à l'humanité la cité-Etat la plus illustre des temps classiques, Athènes. Ces notions sont remarquablement condensées dans cette phrase de Périclès $\varphi \imath \lambda o \kappa \alpha \lambda \circ \hat{v}-$ $\mu \varepsilon v \mu \varepsilon \tau$ ' $\varepsilon \dot{\tau} \tau \varepsilon \lambda \varepsilon i ́ \alpha \varsigma$ prononcée à une époque où l'art - J. Marcadé l'a encore montré récemment ${ }^{5}$ - n'était que le prolongement de la sôphrosynè sur le plan de la création spirituelle, tandis que celui des temps hellénistiques se caractérise par l'hybris dont l'apparition correspond à la disparition de la cité-Etat. Création artistique, comportement social, technique de la guerre et religion officielle des temps classiques reposent sur la conception de la sôphrosyne, considérée par les thesmothètes et les tyrans de l'époque archaïque comme la condition fondamentale pour la création d'un Etat fort et prospère. A Athènes, à Sparte, à Corinthe, à Sicyone, à Milet, presque partout dans le monde grec, l'apparition de la cité-Etat est étroitement liée à l'oeuvre des thesmothètes ou aisymnètes chargés' de mettre fin aux troubles internes des cités et de restaurer la paix.

Presque toujours, écrit G. Glotz ${ }^{6}$, c'est en pleine guerre civile, au milieu des passions déchaînées, que travaillait le législateur. Il ne s'agissait pas pour lui de faire à tête reposée une oeuvre de cabinet, mais d'arrêter l'effusion de sang par une conciliation. Il était désigné par un compromis pour intervenir comme arbitre entre les factions frémissantes. Investi de pouvoirs extraordinaires, il devenait pour tout le temps nécessaire le chef suprême de la cité.

C'est pourquoi nombre de ces législateurs, comme Solon d'Athènes et Périandre de Corinthe, furent appelés «sages», qualificatif donné également à Lycurgue de Sparte par Plutarque ${ }^{7}$. Comme l'a encore souligné tout récemment N. Papachatzis ${ }^{8}$, cette appellation ne doit pas nous faire croire à des individus doués de connaissances supérieures ou à un genre de pré-philosophes; elle désigne des hommes de bon sens, au jugement avisé et juste, oeuvrant dans le dessein d'appliquer la sôphrosynè à tous les niveaux de la vie quoti-

5 J. MARCADÉ, Le classicisme dans la sculpture grecque du IVe siècle, in XIIe Congrès international d'Archéologie classique. Athènes, 4-10 septembre 1983, (Actes sous presse).

6 GLOTZ, op. cit., p. 116-117.

7 PLUT., Vit, Lyc., 30.

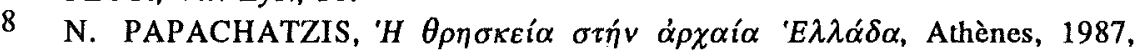
p. 99-101. 
dienne. Ainsi Lycurgue a établi un équilibre social garant de l'ordre et de la paix au sein d'une foule dont le comportement est caractérisé par l'hybris ${ }^{9}$, en prenant des mesures extrêmes qui visaient à supprimer toute forme d'excès et de luxe dans la vie sociale ${ }^{10}$, usages funéraires ${ }^{11}$ et expression artistique ${ }^{12}$ compris. La politique de Solon, qui s'était fait seconder, selon une tradition, par un autre sage, Epiménide de Crète ${ }^{13}$, était animée de cette même volonté d'abolir tout excès : il défendit, par exemple, que l'on s'attaque aux morts, afin que les haines ne fussent pas éternelles, et que l'on injurie les vivants dans les sanctuaires et autres lieux publics; il fixa des limites rigoureuses aux donations, réprima le désordre et la licence des sorties des femmes, interdit les sacrifices funéraires luxueux ainsi que les gestes de lamentation exagérés ${ }^{14}$. «La plupart de ces défenses subsistent encore dans nos lois» atteste Plutarque ${ }^{15}$. L'intervention vigoureuse de cette législation dans toutes les manifestations de la vie sociale justifie sa qualification de «tyrannie élective» par Aristote ou de «dictature» par Denys d'Halicarnasse ${ }^{16}$.

La sôphrosyne commandait aussi le comportement du soldat, désormais intégré dans la formation groupée qui est la «condition militaire» d'existence de la cité-Etat, c'est-à-dire la phalange, dont la plus ancienne représentation connue figure sur l'olpè Chigi (milieu du VIIe s. av. J.-C.). Au contraire du combat singulier où rivalisaient d'exploits individuels deux champions emportés par un sentiment d'exaltation proche de la folie furieuse, cette nouvelle forme de combat exigeait des hoplites de se soumettre à une même discipline, de rythmer et de coordonner leurs mouvements pour que la phalange se déplace comme un seul homme. A ce propos, $M$. Detienne s'est exprimé en ces termes ${ }^{17}$ :

9 PLUT., Vit. Lyc., 5.

10 Ibid., 9.

11 Ibid., 27.

12 Ibid., 9.

13 PLUT., Vit. Solon., 12, 7.

14 Ibid., 21.

15 Ibid., 21, 7 (coll. des Universités de France).

16 ARISTOT., Polit., III, 9 (14), 5; DION. HAL., 5, 73. Cf. GLOTZ, op. cit., p. 117.

17 M. DETIENNE, La phalange : problèmes et controverses, in J.-P. VERNANT (éd.), Problèmes de la guerre en Grèce ancienne, Paris, 1968, p. 119-142, et précisément p. 120. 
Entre le sens technique de position occupée par l'hoplite et les valeurs éthiques de maitrise de soi, de discipline et d'ordre, il n'y a pas d'hiatus. Sur le plan des conduites, le changement est donc radical : la $\sigma \omega \varphi \rho o \sigma u ́ v \eta$, la «maîtrisc entière de soi», remplace cette ivresse, cette mise hors de soi, qui faisait du guerrier un possédé de Lyssa.

Un changement analogue s'opéra également dans le comportement religieux du citoyen ou, plus exactement, dans le mode d'expression de son sentiment religieux personnel, qui aevait désormais se subordonner aux principes de la «modestie» et de la mesure. L'excès était laissé aux manifestations religieuses officielles organisées par l'Etat ou, très souvent, aux offrandes personnelles des thesmothètes et des tyrans dans les grands sanctuaires panhelléniques. Désireux, selon Aristote ${ }^{18}$, de puiser dans le droit divin une légitimité contestée par le droit humain, les Cypsélides de Corinthe, les Orthagorides de Sicyone et les tyrans de la Sicile rivalisaient de luxe dans leurs offrandes à Delphes et à Olympie.

A Athènes, il paraît que Solon, sur les conseils d'Epiménide, avait essayé d'imposer la modération à toute une série de manifestations religieuses privées, comme il l'avait fait pour les cérémonies funéraires : aux dires de Plutarque ${ }^{19}$,

il accoutuma les Athéniens à plus de simplicité dans les manifestations de deuil, incorporant aussitôt certains sacrifices aux funérailles et en supprimant des pratiques rudes et barbares auxquelles la plupart des femmes s'astreignaient auparavant.

C'est une sévère leçon de simplicité que le sage crétois donna aux Athéniens lorsqu'il préféra, aux richesses considérables que les citoyens voulaient lui offrir pour le remercier de son concours, une branche de l'olivier sacré d'Athènes ${ }^{20}$. La forme définitive de la religion d'Athènes, celle que nous lui connaissons aux temps classiques, lui fut imprimée par Pisistrate. Selon A. Andrewes ${ }^{21}$, la politique religieuse du tyran fut d'une importance considérable pour l'organisation sociale et politique de la ville qui deviendra au siècle suivant la cité-Etat la plus prospère du monde antique. Pour

18 ARISTOT., Polit., VIII (V), 9, 15.

19 PLUT., Vit. Solon., 12, 8 (coll. des Universités de France).

20 Ibid., 12, 12.

21 A. ANDREWES, The Greek Tyrants, Londres, 1956, p. 113-115. 
G. Glotz ${ }^{22}$, les réformes de Pisistrate, comme celles des tyrans des autres villes archaïques, visaient à la création d'une sorte de panthéon «national» composé de divinités à la fois panhelléniques et poliades. Parmi ces dernières ne figuraient pas celles au caractère aristocratique affirmé mais, au contraire, les dieux populaires et les héros agrestes qui reçurent des honneurs spéciaux, surtout quand ils étaient en rapport avec la localité d'où était issue la famille du tyran et avaient ainsi un aspect dynastique en même temps que démocratique ${ }^{23}$.

L'opinion d'A. Andrewes est un peu plus complète 24 : hormis leur caractère anti-aristocratique, les cultes propagés par les tyrans, et surtout par Pisistrate, avaient pour foyer la cité-Etat. Dans le programme religieux de Pisistrate, nous pouvons distinguer deux volets. L'objet du premier était d'accroître et d'officialiser le culte des divinités que le mythe et les croyances populaires attachaient directement à «l'histoire nationale» de la ville d'Athènes d'une part, et, de l'autre, au progrès social et à la notion de vigueur de l'Etat. Thésée, aux exploits duquel Athènes devait son indépendance de la Crète minoenne (on se souviendra des représentations de la légende du Minotaure dans la céramique attique du milieu du VIe siècle, dont les exemples les plus fameux sont le cratère François et la coupe $n^{\circ} 2243$ de Munich ${ }^{25}$ ) et son assainissement des phénomènes sociaux pathogènes (comme la piraterie et le brigandage), Thésée donc, fut reconnu comme une sorte de héros national. Et cela en dépit du fait que l'oikos des Pisistratides, comme l'a soutenu J. Boardman ${ }^{26}$, marquait une préférence envers le héros «dorien» Héraclès qui s'était toutefois également signalé en tant que bienfaiteur pour l'espèce humaine : il serait superflu de rappeler ici les exploits d'Héraclès tenus pour de grands travaux agricoles ou autres.

C'est un caractère (semi ?-) officiel qu'ont acquis les Mystères de Déméter, la déesse qui a extrait les hommes du monde animal en leur offrant un mode de vie civilisé, célébrés dans son sanctuaire

22 GLOTZ, op. cit., p. 121-122.

23 Ibid., p. 121.

24 ANDREWES, op. cit., p. 114.

25 Cf. H. CASSIMATIS, s.v. Athèna, in LIMC, II(1984), p. 1006, no 536

26 J. BOARDMAN, Herakles, Peisistratos and Sons, in RA (1972), p. 57-72. 
d'Eleusis qu'Athènes contrôlait depuis l'époque de Solon ${ }^{27}$; Pisistrate y a aussi fait construire une nouvelle salle d'initiation, ledit telesterion, dont le rythme et les dimensions monumentales autorisent à qualifier d'austère l'édifice antérieur élevé probiıblement sous Solon. Le plan du telesterion du tyran fut adopté par les grands chefs de la démocratie athénienne du Ve siècle, tels Cimon et Périclès, qui se sont limités à en développer la surface ${ }^{28}$. Il semble que c'est le culte d'Athéna, la déesse poliade d'Athènes, qui fut le plus intensifié : la célébration des Grandes Panathénées, instaurées quelques années avant l'arrivée au pouvoir de Pisistrate par l'archonte Hippokleides (566 av. J.-C.), a commencé à s'identifier à une manifestation de majesté politique de la part de la ville d'Athènes. Avec les Pisistratides, l'Etat d'Athènes honore et se consacre à la divinité qui était en quelque sorte la «personnification divine» de la notion de sôphrosyne. Détentrice absolue de la sagesse, Athéna sera représentée dans la céramique attique depuis le dernier quart du VIe siècle en tant qu'ergane ${ }^{29}$, tandis que les poèmes homériques et les oeuvres littéraires archaïques l'avaient déjà chantée maîtresse de la technè, de la créativité, condition fondamentale pour l'élévation du niveau de vie des hommes ${ }^{30}$. C'est sous la tyrannie de Pisistrate qu'Athéna remportera définitivement la victoire sur certains représentants de l'ancienne religion caractéristiques d'une société sans justice sociale, d'une société où l'individu était d'abord «homo religiosus» puis seulement citoyen : bien que dans les textes littéraires la déesse l'eût emporté sur son homologue de l'ancienne religion, Gorgô, à partir du milieu du VIIIe siècle ${ }^{31}$, et que dans l'art attique elle ait été figurée assistant à la décapitation de Méduse par son protégé Persée à partir du milieu du VIIe siècle ${ }^{32}$, elle ne s'appropria le gorgoneion que sous Pisistrate : M. Halm-Tisserant y

27 Cf. notamment G. MYLONAS, Eleusis and the Eleusinian Mysteries, Princeton, 1961 (1974), p. 8, 67-70.

28 Cf. MYLONAS, op. cit., p. 55-154, fig. 6, 25-27.

29 Cf. P. DEMARGNE, s.v. Athèna, in LIMC, П(1984), p. 962, n 40 (coupe attique $\mathrm{n}^{\circ}$ inv. 166 du musée de l'Acropole d'Athènes).

30 Cf. les sources rassemblées par DEMARGNE, ibid., p. 961.

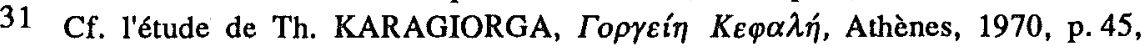
91, 101.

32 Cf. H. CASSIMATIS, s.v. Athèna, in LIMC, II(1984), p. 1003, n 504 (coupe attique $\mathrm{n}^{\circ}$ inv. B 380 du British Museum). 
voit «une récupération à des fins de propagande, une pratique connue et admise pour d'autres thèmes» ${ }^{33}$ comme la gigantomachie (le combat des dieux olympiens représentant l'harmonie cosmique contre les démons d'une société primitive) et les exploits d'Héraclès.

Assurément, le dieu Apollon, le pendant masculin d'Athéna en sagesse et en mesure, ne pouvait manquer de figurer dans le cadre d'un tel programme religieux. Bien que son oracle à Delphes, dont l'objectif essentiel était déjà aux temps archaïques d'établir l'ordre dans les cités grecques, subît la forte influence des ennemis politiques de Pisistrate, les Alcméonides, le tyran athénien ne négligea pas de fonder un temple en l'honneur d'Apollon Patrôos, le dieu ancestral des Ioniens. L'endroit choisi pour la construction de son sanctuaire n'est pas dépourvu de signification : le temple d'Apollon fut érigé sur l'agora ${ }^{34}$, et $\mathrm{R}$. Martin a souligné le rôle essentiel de ce lieu dans le programme politique de Pisistrate pour la conquête et la conservation du pouvoir ${ }^{35}$. Le tyran a également favorisé l'orphisme, courant de pensée religieuse apollinienne par excellence. L'importance accrue de l'orphisme sous la tyrannie de Pisistrate et de ses fils - hormis certaines théories qui l'attribuent aux liens de parenté qui unissaient le tyran et la famille royale mycénienne de Pylos tenue pour le berceau préhistorique de la secte en question ${ }^{36}$ doit être également envisagée dans le cadre de l'application de la sôphrosynè dans le rituel. M. Detienne ${ }^{37}$ a judicieusement observé que le rituel orphique devient parfaitement compréhensible uniquement dans sa confrontation avec le drômenon dionysiaque qui aidé l'individu à s'échapper de sa «condition politico-religieuse» par un rituel orgiastique de possession reposant sur le principe de l'ômophagie, signe par excellence de la bestialité, précisément à

33 M. HALM-TISSERANT, Le Gorgoneion, emblème d'Athéna. Introduction du motif sur le bouclier et l'égide, in RA (1986), p. 245-278, plus spécialement p. 277. Sur le sujet, cf. aussi l'étude de N. PAPACHATZIS, L'origine de la déesse Athéna : un réexamen de l'évidence, ici même.

34 Cf. entre autres E. SIMON, Festivals of Attica. An Archaeological Commentary, Wisconsin, 1983, p. 74.

35 R. MARTIN, Recherches sur l'agora grecque, Paris, 1951, p. 270-271.

36 Cf. par exemple R. BOEHME, Orpheus der Sänger und seine Zeit, BerneMunich, 1970, p. 314-318.

37 M. DETIENNE, Pratiques culinaires et esprit de sacrifice, in M. DETIENNE - J. P. VERNANT (éd.), La cuisine du sacrifice èn pays grec, Paris, 1979, p. 7-35, plus spécialement p. 16. 
l'opposé du comportement circonspect et réfléchi, fondement d'une société organisée et structurée.

L'attitude de Pisistrate à l'égard du dionysisme constitue le second volet bien distinct de son programme religieux. Comme les tyrans de Sicyone et de Naxos, Pisistrate encouragea le culte populaire de Dionysos dans le dessein de se joindre l'appui des milieux principalement ruraux. La céramique attique témoigne de la faveur de Dionysos et de ses acolytes démoniaques, tels satyres et ménades, au VIe siècle : le cratère François associe le dieu au thème du «Retour d'Héphaistos», un des sujets les plus prisés des cortèges populaires pré-dramatiques dionysiaques ${ }^{38}$, et sur la coupe $n^{\circ} 5179$ de Copenhàgue ( $c a 560$ av. J.-C.) Bacchus est accompagné de satyres et de ménades ${ }^{39}$. C'est dans cette intention que Pisistrate a promu les festivités dionysiaques connues par les générations postérieures sous le nom de Grandes et Petites Dionysies. Cependant, le drômenon bacchique, de caractère orgiastique, avait une fonction psychologique qui allait à l'encontre des qualités attendues du nouveau type de citoyen dont avait besoin la cité-Etat : comme l'a signalé E. Dodds, la danse frénétique et le drômenon de possession par la mania divine envoyée par Dionysos visaient précisément à satisfaire l'inclination de l'individu à rejeter le sentiment de responsabilité et toutes les contraintes qu'exerce la société ${ }^{40}$. C'est la raison pour laquelle d'ailleurs Platon, dans ses Lois, condamne le drômenon bacchique en tant que ov $\pi 0 \lambda_{\imath \imath \imath \text { cóv }}{ }^{41}$. Nous ignorons comment Pisistrate réussit le tour de force qui consistait d'une part à encourager le culte dionysiaque, et de l'autre à exclure l'élément orgiastique. Certes l'orgiasme était toujours présent dans le dionysisme, même aux temps classiques, et cela sans nuire à l'idéal du citoyen modèle tenu à se comporter sagement dans le cadre du fonctionnement de l'Etat et à assumer ses responsabilités sociales et politiques. Il semble, par contre, que le dionysisme, en donnant la possibilité au citoyen de se sentir affranchi de toute contrainte sociale à l'occasion - et le temps d'une fête religieuse, lui permettait ainsi de conserver un équilibre

38 Pour le thème du «retour d'Héphaistos», cf. entre autres F. BROMMER, Die Rückführung des Hephaistos, in JdI, 52(1937), p. 198-219; ID., Hephaistos, Mainz, 1978; Th. CARPENTER, Dionysian Imagery in Archaic Greek Art, Oxford, 1986, chap. II.

39 Cf. CARPENTER, op. cit., p. 83.

40 E. DODDS, The Greeks and the Irrational, Berkeley, 1951, chap. III.

41 PLAT., Leg., $815 \mathrm{c}-\mathrm{d}$. 
psychologique si nécessaire pour sa fonction de $\pi 0 \lambda$ ı 1 kòv öv. Néanmoins il est aussi vrai qu'à l'époque classique certains aspects du dionysisme avaient perdu le caractère orgiastique qu'ils avaient eu en des temps plus archaïques.

E. Dodds a attiré l'attention sur le' fait qu'une fois le rituel dionysiaque incorporé dans la religion de l'Etat, Dionysos n'était plus couramment considéré comme le dieu par excellence de l'extase orgiastique. Bacchus est absent du passage de l'Hippolyte

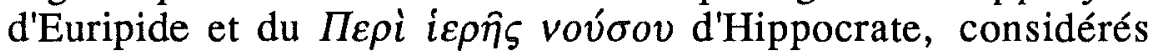
comme deux catalogues de ces divinités que la pensée de la fin du Ve siècle associait aux bouleversements psychosomatiques provoqués par l'extase rituelle ${ }^{42}$. Mais il est un domaine à propos duquel les Athéniens des temps classiques se plaignaient de ce qu' «il n'y avait rien pour Dionysos» : c'est celui de la création dramatique devenue totalement étrangère à son point de départ qui était le spectacle religieux dionysiaque. C'est à l'époque de Pisistrate, ou peu avant, que la tradition situait la transformation des kômoi orgiastiques prédramatiques en représentations théâtrales qui n'étaient plus célébrées dans la campagne. Ces manifestations se tenaient désormais au centre de la cité, en un lieu qui, de par sa distribution de l'espace et sa forme architecturale, était identique à l'édifice où se prenaient les décisions politiques : le théâtre. Sous Pisistrate, le théâtre, monté sur gradins mobiles, était élevé à l'agora même, qui devenait déjà le coeur de la vie politique et religieuse d'Athènes. En accordant un prix aux représentations pré-dramatiques dionysiaques, le pouvoir politique leur conféra l'aspect de l'agôn, rouage du système de la cité-Etat perceptible dans ses diverses manifestations, la guerre y compris : au Vîe siècle, à l'individu combattant sous l'effet de l'enthousiasme et de la lyssa, a été substituée la phalange (cf. supra) et ses mouvements comme l'attitude des hoplites qui la constituaient étaient réglés suivant des principes agonistiques qui participent de la sôphrosyne. Sous Pisistrate, la conception agonistique est imposée dans le domaine de la célébration religieuse dionysiaque qui se transforma rapidement en ce qu'on appelle aujourd'hui «théâtre classique» regardé comme la clef de voûte spirituelle de la cité-Etat athénienne.

42 EUR., Hippol., 141 sq.; HIPPOKR., Morb. Sacr., I, VI. 360. 13 sq. 
Selon la tradition, le premier genre de théâtre classique issu des représentations des kômoi dionysiaques fut la comédie : Sousarion, celui qui remporta le prix du premier agôn dramatique, est considéré

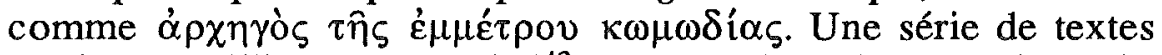
tardifs recueillis par G. Kaibe ${ }^{43}$ se font le lointain écho d'un substrat politique à la source de la transformation des kômoi pré-dramatiques en agôn théâtral : il semble que l'Etat, en accordant des prix, voulait encourager des «poètes» à dénoncer, au travers de leurs pièces, les injustices que commettaient les riches citoyens de la ville envers les paysans athéniens, qui, jusqu'alors, s'en chargeaient euxmêmes : rentrés la nuit à l'ö $\sigma \tau v$, ils provoquaient du désordre en allant crier aux portes de leurs offenseurs les injustices dont ils avaient été victimes.

Thisseos 45, Chalandri,

Ioannis LOUCAS GR - 15234 ATHÈNES

43 G. KAIBEL, $C G F, 12$ sq. Cf. A. PICKARD-CAMBRIDGE, Dithyramb, Tragedy and Comedy2, Oxford, 1962, p. 184. 